\title{
METALLIC NANOPARTICLES INFLUENCE ON BLOOD FLOW THROUGH A STENOTIC ARTERY
}

\author{
Khaled S. Mekheimer ${ }^{1}$, Mohamed S. Mohamed ${ }^{2}$, Thanaa Elnaqeeb ${ }^{3}$ \\ ${ }^{1,2}$ Department of Mathematics and Statistics \\ Faculty of Science \\ Taif University \\ Hawia 888, Taif, SAUDI ARABIA \\ ${ }^{1,2}$ Department of of Mathematics \\ Faculty of Science \\ Al-Azhar University \\ Nasr City, Cairo, EGYPT \\ ${ }^{3}$ Department of Mathematics \\ Faculty of Science \\ Zagazig University \\ Zagazig, EGYPT
}

\begin{abstract}
A model of magnetohydrodynamic blood flow through an artery with a mild stenosis under the influence of metallic nanoparticles is presented. The coupled nonlinear partial differential equations governing the problem are simplified by subject to reasonable modeling and stenosis approximations to obtain analytic expressions for the flow characteristics. The expressions for velocity, temperature, stream function, flow resistance and wall shear stress distribution are obtained in the present analysis. Effects of some emerging physical parameters on arterial blood flow characteristics are shown graphically and discussed briefly under the influence of metallic nano particles (nanofluid model). Finally, stream line patterns illustrating size and number of trapping boluses are introduced and discussed for various imbedded parameters.
\end{abstract}

Key Words: blood flow, arteries, metallic nanoparticles, overlapping stenosis, MHD

Received: November 27, 2015

Published: April 6, 2016

§ Correspondence author (c) 2016 Academic Publications, Ltd. url: www.acadpubl.eu 


\section{Introduction}

Stenoses in the arteries of mammals are common occurrence, and for many years researchers have endeavored to model the flow of blood through stenosed arteries experimentally and theoretically.

It is known that arterial diseases are chief cause of death in most of the western world. Although the genesis of such diseases remains unknown, there is a strong belief that hydrodynamic factors play a very significant role in the formation and proliferation of the disease.

The deposition of cholesterol and proliferation of the connective tissues in the arterial wall form plaques which grow inward and restrict blood flow. Stenosis or arteriosclerosis, which means narrowing of any body passage (tube or orifice), is thus an abnormal and unnatural increase in the arterial wall thickness that develops at various locations of the cardiovascular system under diseased conditions.

In order to have a fuller understanding of the development of these diseases, an accurate knowledge of the mechanical properties of the vascular wall together with the flow characteristics of blood are indispensable. Thus, the relevant information is deemed to be of great help in the treatment of vascular diseases and also to bioengineers who are engaged in the design and construction of improved artificial organs. Perhaps the actual cause of abnormal growth in an artery is not completely clear to the theoretical investigators but its effect over the cardiovascular system has been determined by studying the flow characteristics of blood in the stenosed area.

Although the applicability of a purely mechanical model for such a physiological problem has obvious limitations, vascular rheology together with hemodynamic factors are predominant in the development and progression of arterial stenosis, see [1]. Recently, Mekheimer et al (see [2]) study the effect of the induced magnetic field on blood flow through an anisotropically tapered elastic arteries with overlapping stenosis in an annulus.

If a magnetic field is applied to a moving electrically conducting liquid, it induces electric and magnetic fields. The interaction of these fields produces a body force known as Lorentz force which has a tendency to oppose the movement of the liquid (see [3]), Stud et al [4] studied the effect of moving magnetic field on blood flow and observed that the effect of suitable moving magnetic field accelerates the speed of blood. Agrawal and Anwaruddin in [5] studied the effect of a magnetic field on blood flow by taking a simple mathematical model for blood through an equally-branched channel with flexible walls executing peristaltic waves using a long wavelength approximation method and observed, 
for the flow of blood in arteries with arterial disease like arterial stenosis or arteriosclerosis, that the influence of magnetic field may be utilized as a blood pump in carrying out cardiac operations. The problem of biomechanics has attracted the attention of many investigation, see [6-8]. The important contributions of recent years to the topic are referenced in the literature, see [9-18]. Theoretical investigations have been carried out to study the effect of multi-stenosis in the presence of a magnetic field, see [19-22].

Nanofluids have been found possess to enhanced thermo physical properties such as increased thermal diffusivity, thermal conductivity, convective heat transfer coefficients and viscosity as compared to those of base fluids like oil, water or blood. The most important distinguishing of nanofluids is their high thermal conductivity relative to the base fluids. The theory of nanofluid was developed by Choi in [23] and after him highlighted by others, see [24-25]. Recently, many researchers studied the effect of nanoparticles on some models for the flow of physiological fluids, such as, blood flow and peristaltic flow, see [26-31].

In this paper, we study the effects of metallic nanoparticles on the blood flow (conducting fluid) through an elastic vertical artery with a stenosis under effect of an external magnetic field. Such analysis may be useful in understanding magnetic resonance angiography (MRA) which is one of radiological investigations of atherosclerosis. The study is also useful for evaluating the role of nanoparticles of the stenosed arterial wall when the body is subjected to magnetic resonance imaging (MRI).

\section{Formulation and Analysis of the Physical Problem}

The equations, which govern the steady flow of an incompressible viscous and electrically conducting nanofluid in the presence of a magnetic field, are:

$$
\begin{aligned}
& \boldsymbol{\nabla} \cdot \boldsymbol{V}=0, \\
& \rho_{n f}(\boldsymbol{V} \cdot \boldsymbol{\nabla} \boldsymbol{V})=-\boldsymbol{\nabla} p+\mu_{n f} \nabla^{2} \boldsymbol{V}+\boldsymbol{J} \times \boldsymbol{B}+\boldsymbol{g}(\rho \gamma)_{n f}\left(T-T_{1}\right), \\
& \left(\rho c_{p}\right)_{n f} \boldsymbol{V} \cdot \boldsymbol{\nabla} T=K_{n f} \boldsymbol{\nabla}^{2} T+Q_{0}, \\
& \boldsymbol{\nabla} \times \boldsymbol{B}=\mu_{e} \boldsymbol{J}, \quad \boldsymbol{\nabla} \cdot \boldsymbol{B}=0, \quad \boldsymbol{\nabla} \times \boldsymbol{E}=0, \quad \boldsymbol{\nabla} . \boldsymbol{J}=0 .
\end{aligned}
$$

The generalized Ohm's law taking the Hall effects into account is

$$
\boldsymbol{J}=\sigma[\boldsymbol{E}+\boldsymbol{V} \times \boldsymbol{B}]-\frac{\sigma}{e n_{e}} \boldsymbol{J} \times \boldsymbol{B} .
$$


In writing equation (5), the ion slip and the thermo-electric effects are neglected. Here $\boldsymbol{B}$ is the total magnetic induction vector, $\boldsymbol{V}$ is the velocity vector $\boldsymbol{E}$ is the electric field vector, $\boldsymbol{J}$ is the current density vector; $\sigma$ is the electrical conductivity of the fluid, $e$ is the electric charge, $n_{e}$ is the number density of electrons, $\mu_{e}$ is the magnetic permeability, $T$ as the temperature of fluid, $Q_{0}$ as constant heat absorption or heat generation, $\mu_{n f}$ is the nanofluid viscosity, $K_{n f}$ is the thermal conductivity of nanofluid, $\rho_{n f}$ is the nanofluid density, $\left(\rho c_{p}\right)_{n f}$ is the heat capacitance of nanofluid and $(\rho \gamma)_{n f}$ is the thermal expansion coefficient. The heat transfer is taken into account by giving temperature $T_{1}$ to the upper wall of the tube.

It is assumed that blood flow is represented by an incompressible viscous nanofluid flowing through a tube of length $L$ with overlapping stenosis (see Figure 1). Let $z$ and $r$ axes be chosen along and perpendicular to the walls, respectively. A uniform magnetic field $B_{0}$ is acting along the $r$-axis (i.e. $B_{0}$ is applied in a direction perpendicular to the flow of the fluid).

The geometry of the stenotic arterial wall

$$
h(z)=\left\{\begin{aligned}
R_{0}-\frac{\delta(z-d)}{31-\frac{94}{3 L_{0}}(z-d)} & \\
\left.\quad+\frac{32}{L_{0}^{2}}(z-d)^{2}-\frac{32}{3 L_{0}^{3}}(z-d)^{3}\right], & d \leq z \leq d+\frac{3}{2} L_{0}, \\
R_{0}, & \text { otherwise, }
\end{aligned}\right.
$$

where the onset of the stenosis is located at a distance $\mathrm{d}$ from the inlet, $\frac{3 L_{0}}{2}$ the length of the overlapping stenosis and $L_{0}$ representing the distance between two critical height of the stenoses.

The thermo-physical properties as given in [30] and [31] are

$$
\begin{aligned}
\mu_{n f} & =\frac{\mu_{f}}{(1-\Phi)^{2.5}}, \\
\alpha_{n f} & =\frac{K_{n f}}{\left(\rho c_{p}\right)_{n f}}, \\
\rho_{n f} & =(1-\Phi) \rho_{f}+\phi \rho_{s}, \\
(\rho \gamma)_{n f} & =(1-\Phi)(\rho \gamma)_{f}+\Phi(\rho \gamma)_{s}, \\
\left(\rho c_{p}\right)_{n f} & =(1-\Phi)\left(\rho c_{p}\right)_{f}+\Phi\left(\rho c_{p}\right)_{s}, \\
\frac{K_{n f}}{K_{f}} & =\frac{\left(K_{s}+2 K_{f}\right)-2 \Phi\left(K_{f}-K_{s}\right)}{\left(K_{s}+2 K_{f}\right)+\Phi\left(K_{f}-K_{s}\right)},
\end{aligned}
$$




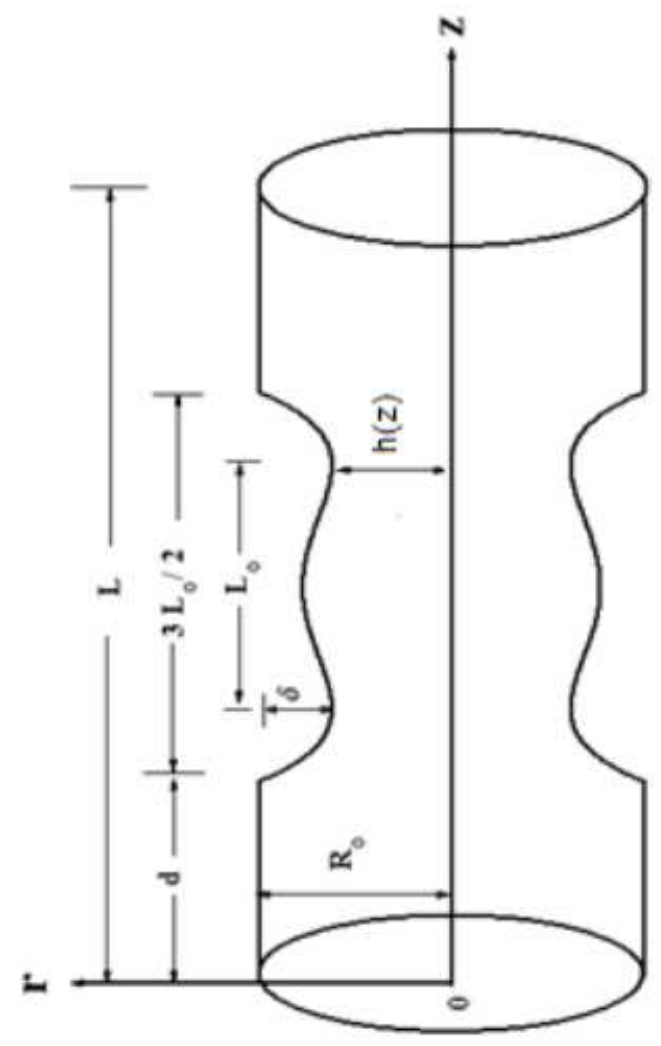

Figure 1: Schematic diagram of an arterial overlapping stenosis

where $\rho_{f}, \mu_{f}, \gamma_{f},\left(\rho c_{p}\right)_{f}$, and $K_{f}$ are the density, viscosity, the thermal expansion coefficient, the heat capacitance and the thermal conductivity of the base fluid, respectively. While $\rho_{s}, \mu_{s}, \gamma_{s},\left(\rho c_{p}\right)_{s}$, and $K_{s}$ are the density, viscosity, the thermal expansion coefficient, the heat capacitance and the thermal conductivity of the metallic nanoparticle, respectively and $\Phi$ is the volume fraction.

Then the governing equations for conservation of mass, momentum and temperature for a viscous conducting nanofluid can be written as

$$
\frac{\partial u^{\prime}}{\partial r^{\prime}}+\frac{\partial w^{\prime}}{\partial z^{\prime}}+\frac{u^{\prime}}{r^{\prime}}=0
$$




$$
\begin{gathered}
\rho_{n f}\left(u^{\prime} \frac{\partial w^{\prime}}{\partial r^{\prime}}+w^{\prime} \frac{\partial w^{\prime}}{\partial z^{\prime}}\right) \\
=-\frac{\partial P^{\prime}}{\partial z^{\prime}}+\mu_{n f}\left[\frac{\partial^{2} w^{\prime}}{\partial r^{\prime 2}}+\frac{1}{r^{\prime}} \frac{\partial w^{\prime}}{\partial r^{\prime}}+\frac{\partial^{2} w^{\prime}}{\partial z^{\prime 2}}\right]+g(\rho \gamma)_{n f}\left(T^{\prime}-T_{1}^{\prime}\right)-\sigma B_{0}^{2} w^{\prime}, \\
\rho_{n f}\left(u^{\prime} \frac{\partial u^{\prime}}{\partial r^{\prime}}+w^{\prime} \frac{\partial u^{\prime}}{\partial z^{\prime}}\right)=-\frac{\partial P^{\prime}}{\partial r^{\prime}}+\mu_{n f}\left[\frac{\partial^{2} u^{\prime}}{\partial r^{\prime 2}}+\frac{1}{r^{\prime}} \frac{\partial u^{\prime}}{\partial r^{\prime}}-\frac{u^{\prime 2}}{r^{\prime 2}}+\frac{\partial^{2} u^{\prime}}{\partial z^{\prime 2}}\right], \\
u^{\prime} \frac{\partial T^{\prime}}{\partial r^{\prime}}+w^{\prime} \frac{\partial T^{\prime}}{\partial z^{\prime}}=\frac{K_{n f}}{\left(\rho c_{p}\right)_{n f}}\left[\frac{\partial^{2} T^{\prime}}{\partial r^{\prime 2}}+\frac{1}{r^{\prime}} \frac{\partial T^{\prime}}{\partial r^{\prime}}+\frac{\partial^{2} T^{\prime}}{\partial z^{\prime 2}}\right]+\frac{Q_{0}}{\left(\rho c_{p}\right)_{n f}} .
\end{gathered}
$$

Here $u^{\prime}$ and $w^{\prime}$ are the velocity components in the radial and axial directions. For the proposed nanofluid model $\mu_{n f}$ is the viscosity, $K_{n f}$ as thermal conductivity, $\rho_{n f}$ as density, $K_{n f}$ as thermal expansion coefficient and $\left(c_{p}\right)_{n f}$ as heat capacitance.

We introduce the following non dimensional variables:

$$
\begin{aligned}
& r=\frac{r^{\prime}}{R_{0}}, z=\frac{z^{\prime}}{L_{0}}, p=\frac{R_{0}^{2}}{\mu_{f} u_{0} L_{0}} p^{\prime}, w=\frac{w^{\prime}}{u_{0}}, u=\frac{L_{0} u^{\prime}}{\delta u_{0}}, \\
& R_{e n}=\frac{\rho_{\mathrm{f}} u_{0} L_{0}}{\mu_{\mathrm{f}}}, \delta^{*}=\frac{\delta}{R_{0}}, G_{r}=\frac{g \gamma_{f} \rho_{f} R_{0}^{2} T_{0}}{u_{0} \mu_{f}}, \beta=\frac{Q_{0} R_{0}^{2}}{T_{0} K_{f}}, \\
& \varepsilon=\frac{R_{0}}{L_{0}}, \theta=\frac{T^{\prime}-T_{1}}{T_{0}-T_{1}}, \mathrm{M}=\sqrt{\frac{\sigma}{\mu_{f}}} \mathrm{~B}_{0} L_{0} .
\end{aligned}
$$

Then the governing equations for conservation of mass, momentum and temperature for viscous fluid can be written in the dimensionless variables as:

$$
\begin{array}{r}
\frac{\rho_{n f}}{\rho_{f}} R_{e n} \varepsilon^{2}\left(\delta^{*} u \frac{\partial w}{\partial r}+w \frac{\partial w}{\partial z}\right) \\
=-\frac{\partial P}{\partial z}+\frac{\mu_{n f}}{\mu_{f}}\left[\frac{\partial^{2} w}{\partial r^{2}}+\frac{1}{r} \frac{\partial w}{\partial r}+\varepsilon^{2} \frac{\partial^{2} w}{\partial z^{2}}\right]+\frac{(\rho \gamma)_{n f}}{(\rho \gamma)_{f}} G_{r} \theta-M^{2} w \\
\frac{\rho_{n f}}{\rho_{f}} R_{e n} \varepsilon^{3} \delta^{*}\left(\varepsilon \delta^{*} u \frac{\partial u}{\partial r}+w \frac{\partial u}{\partial z}\right) \\
=-\frac{\partial P}{\partial r}+\frac{\mu_{n f}}{\mu_{f}}\left[\delta^{*} \frac{\partial^{2} u}{\partial r^{2}}+\frac{\delta^{*}}{r} \frac{\partial u}{\partial r}-\delta^{*} \frac{u^{2}}{r^{2}}+\varepsilon \frac{\partial^{2} u}{\partial z^{2}}\right],
\end{array}
$$




$$
\begin{aligned}
& R_{e n} P_{r n} \varepsilon^{2}\left(\delta^{*} u \frac{\partial \theta}{\partial r}+w \frac{\partial \theta}{\partial z}\right) \\
& =\frac{K_{n f}\left(\rho c_{p}\right)_{f}}{K_{f}\left(\rho c_{p}\right)_{n f}}\left[\frac{\partial^{2} \theta}{\partial r^{2}}+\frac{1}{r} \frac{\partial \theta}{\partial r}+\varepsilon^{2} \frac{\partial^{2} \theta}{\partial z^{2}}\right]+\frac{\beta\left(\rho c_{p}\right)_{f}}{\left(\rho c_{p}\right)_{n f}}
\end{aligned}
$$

In the above expressions $\mathrm{G}_{\mathrm{r}}$ is represents as Grashof number, $\mathrm{R}_{\mathrm{en}}$ is the Reynolds number, $\mathrm{p}_{\mathrm{m}}$ is the Prandtl number, $M$ is the Hartmann number, $\beta$ is nondimensional heat source or sink parameter with respect to fluid and $u_{0}$ is the averaged velocity. Using mild stenosis case $\delta^{*} \ll 1$ and $\epsilon \sim O(1)$, the governing equations (13) to (15) can be written as

$$
\begin{gathered}
\frac{\mu_{f}}{\mu_{n f}} \frac{\partial P}{\partial z}=\left[\frac{\partial^{2} w}{\partial r^{2}}+\frac{1}{r} \frac{\partial w}{\partial r}\right]+\frac{\mu_{f}}{\mu_{n f}} \frac{(\rho \gamma)_{n f}}{(\rho \gamma)_{f}} G_{r} \theta-\frac{\mu_{f}}{\mu_{n f}} M^{2} w \\
\frac{\partial P}{\partial r}=0 \\
{\left[\frac{\partial^{2} \theta}{\partial r^{2}}+\frac{1}{r} \frac{\partial \theta}{\partial r}\right]+\beta\left(\frac{\left(K_{s}+2 K_{f}\right)+\Phi\left(K_{f}-K_{s}\right)}{\left(K_{s}+2 K_{f}\right)-2 \Phi\left(K_{f}-K_{s}\right)}\right)=0}
\end{gathered}
$$

The wall equation and the corresponding boundary conditions in the dimensionless form are:

$$
\begin{aligned}
& h(z)= \begin{cases}1-\delta^{*}(z-d)\left(11-\frac{94}{3}(z-d)+32(z-d)^{2}\right. & \\
\left.-\frac{32}{3}(z-d)^{3}\right), & d \leq \mathrm{z} \leq \mathrm{d}+\frac{3}{2} \\
1, & \text { otherwise }\end{cases} \\
& \frac{\partial w}{\partial r}=0, \quad \frac{\partial \theta}{\partial r}=0, \quad \text { at } r=0 \\
& w=0, \quad \theta=0, \quad \text { at } r=h(z) .
\end{aligned}
$$

The exact solutions of equations (16)-(18) and with equation (19) are written as

$$
\begin{gathered}
\theta=-\frac{1}{4} \beta\left(\frac{\left(K_{s}+2 K_{f}\right)+\Phi\left(K_{f}-K_{s}\right)}{\left(K_{s}+2 K_{f}\right)-2 \Phi\left(K_{f}-K_{s}\right)}\right)\left(r^{2}-h^{2}\right), \\
w(r, z)=-\frac{\alpha_{1}}{M^{2}} \frac{\partial p}{\partial z}-\frac{4 G_{r} \alpha \alpha_{2}}{M^{4}}-\frac{G_{r} \alpha \alpha_{2}}{M^{2}}\left(r^{2}-h^{2}\right)+C_{1} I_{0}(M r),
\end{gathered}
$$


where

$$
\begin{aligned}
& C_{1}=\frac{1}{I_{0}(M r)}\left(\frac{\alpha_{1}}{M^{2}} \frac{\partial p}{\partial z}+\frac{4 G_{r} \alpha \alpha_{2}}{M^{4}}\right), \\
& \alpha_{1}=\frac{\mu_{f}}{\mu_{n f}} \\
& \alpha_{2}=\frac{-1}{4} \beta\left(\frac{\left(K_{s}+2 K_{f}\right)+\Phi\left(K_{f}-K_{s}\right)}{\left(K_{s}+2 K_{f}\right)-2 \Phi\left(K_{f}-K_{s}\right)}\right), \\
& \alpha=\frac{\mu_{f}}{\mu_{n f}} \frac{(\rho \gamma)_{n f}}{(\rho \gamma)_{f}} .
\end{aligned}
$$

We can also find the corresponding stream function by using $w=\frac{1}{r} \frac{\partial \psi}{\partial r}$ and $\psi=0$ at $r=0$ then the stream function will be in the form

$$
\psi(r, z)=-\frac{\alpha_{1} r^{2}}{2 M^{2}} \frac{\partial p}{\partial z}-\frac{4 r^{2} G_{r} \alpha \alpha_{2}}{2 M^{4}}-\frac{G_{r} r^{2} \alpha \alpha_{2}}{2 M^{2}}\left(r^{2}-h^{2}\right)+C_{1} r I_{1}(M r)
$$

The volume rate $F(z)$ is given by

$$
F(z)=\int_{0}^{R} r \psi(z, r) d r
$$

Using equations (21) and (22) into equation (24) and after calculating the above integration, we get the expression for pressure gradient in terms of the ow rate as follows:

$$
\frac{\partial p}{\partial z}=-\frac{1}{\frac{\alpha_{1} h}{2}-\frac{\alpha_{1} I_{1}(h M)}{M I_{0}(h M)}}\left(G_{r} \alpha \alpha_{2}\left(\frac{h^{3}}{4}+\frac{4 I_{1}}{M^{3} I_{0}}-\frac{2 h}{M^{2}}\right)-\frac{F M^{2}}{h}\right)
$$

In the above $F$ is constant flow rate and the pressure drop across the length of the mild stenosis is given as

$$
\Delta p=-\int_{0}^{L} \frac{\partial p}{\partial z} d z
$$




\section{Resistance Impedance and Wall Shear Stress}

\subsection{Resistance Impedance}

The resistance to flow (resistance impedance) is obtained from equation (26) as

$$
\lambda=\frac{\Delta p}{F}=\left\{\int_{0}^{d} N(z)_{h=1} d z+\int_{d}^{d+\frac{3}{2}} N(z) d z+\int_{d+\frac{3}{2}}^{L} N(z)_{h=1} d z\right\},
$$

where

$$
N(z)=-\frac{1}{F\left(\frac{\alpha_{1} h}{2}-\frac{\alpha_{1} I_{1}(h M)}{M I_{0}(h M)}\right)}\left(G_{r} \alpha \alpha_{2}\left(\frac{h^{3}}{4}+\frac{4 I_{1}}{M^{3} I_{0}(h M)}-\frac{2 h}{M^{2}}\right)-\frac{F M^{2}}{h}\right) .
$$

To calculate the resistance impedance, the integration included in equation (27) will be calculated numerically.

\subsection{Wall Shear Stress}

Expression for the wall shear stress $\tau_{r}$ is given by (see [7]):

$$
\tau_{R}=-\left(\frac{\partial w}{\partial r}\right)_{r=h} .
$$

\section{Numerical Results and Discussion}

The analytical expressions derived in the present analysis for velocity, wall shear stress distribution at the stenotic region, and the resistance impedance have been numerically computed for different parameters of interest. In the presented study the results are calculated for $\mathrm{Cu}$-blood nanofluid model. The thermo-physical quantities for Specific heat capacity $\left(\rho c_{p}\right)_{s}$, density $\rho_{s}$, thermal conductivity $K_{s}$ and thermal expansion coefficient $\gamma_{s}$ for blood and copper are obtained from [30], [38].

\subsection{Resistance Impedance and Wall Shear Stress}

Figs. (2-6) display the quantitative results of the dimensionless heat source $\beta$, Grashof number (free convection parameter ) $\mathrm{G}_{\mathrm{r}}$, Hartmann number $\mathrm{M}$, the 
stenosis height $\delta$, the nanoparticles volume fraction $\Phi$ and the flow rate $\mathrm{F}$ for the resistance impedance and the wall shear stress. In order to illustrate the metallic nanoparticles effect on the considered problem.

Figs. (2) to (3) show the variation of the resistance impedance to flow along the maximum height of stenosis. It is analyzed that resistance impedance to flow gives larger values for the arteries of the stenosis as comparing to arteries without stenosis $(\delta=0)$. Figure 2 is plotted for different values of the Hartman number $M$ and nanoparticles volume fraction $\Phi$ and found that resistance impedance to flow decreases with an increase in the nanoparticles volume fraction and decreases with an increase in the Hartman number M.

The effects of Grashof number Gr and heat source or sink parameter $\beta$ are given in Figure 3. It is analyzed that resistance impedance to flow decreases with an increase in the values of the Grashof number Gr and heat source or sink parameter $\beta$.

The dependence of the wall shear stress distribution on Grashof number Gr, the Hartmann number M, the stenosis height $\delta$ and the heat source or sink parameter $\beta$ is illustrated in Figs.(4-6). It is analyzed that the wall shear stress increases as the Hartmann number increases and varies inversely with the Grashof number $\mathrm{G}_{\mathrm{r}}$. Figure 5 relates to the variation of wall shear stress for different values of stenosis height $\delta$ against the axial distance $\mathrm{x}$. It is illustrated from this graph that stress on the wall of arteries is directly proportional to the stenosis height $\delta$, and as if we increase the stenosis height the stress on wall will acquire the higher amplitude. Also from this figure it is analyzed that stresses on wall of artery decreases with an increase in heat source $\beta$ due to contribution of heat to the flow region from the wall of the artery.

Figure 6 is plotted for different values of nanoparticles volume fraction $\Phi$ and flow rate F. we can see that the stresses on the wall of artery decreases with an increase in concentration of nanoparticles and this effect is significant for a small values of the flow rate.

\subsection{Velocity and Temperature Distribution}

The variations of velocity and temperature profile for different values of stenosis height $\delta$; nanoparticle volume fraction $\Phi$; heat source or sink parameter $\beta ; \mathrm{G}_{\mathrm{r}}$ and the Hartmann number $\mathrm{M}$ are shown in Figs. (7) to (10). Figs. (7) and (8) are plotted to observe that how the axial velocity $\mathrm{u}$ is influenced the blood flow through stenosis. Maximum velocity occurs at zero (centre of the artery) and starts decaying to the wall of the artery. From Figs. (7); we observed that velocity profile increases with an increase in the values of Grashof number Gr at 
the center of the artery and this variation is very slowly near the artery walls, which physically means an increase in the viscous forces near the wall of the artery. The variations of temperature profile for heat source or sink parameter $\beta$ is given in Figure 9 and observed that the temperature profile increases with an increase in the values of heat source or sink parameter $\beta$ because of increase in the thermal state of the fluid. From Figure 10, it is analyzed that temperature profile decreases with an increase in the concentration of nanoparticles. The temperature profile for different values stenosis height $\delta$ is given in Figs. (910). It is observed from these graphs that temperature profile increases with an increase in the values of stenosis height $\delta$.

\subsection{Trapping Phenomena}

Trapping characterized an interesting phenomenon for the blood flow in an stenosed artery that is discussed in Figs. (11) to (13). From these figures, it is depicted that size of trapping bolus decreases with an increase in Grashof number $\mathrm{Gr}$ and heat source or sink parameter $\beta$. The effects of the flow rate $\mathrm{F}$ is discussed in Figure 13 and it's shown that the size of the trapping increases as the flow rate increases.

\section{Acknowledgments}

This paper was funded by the Deanship of Scientific Research (DSR), Taif University (TU), under grant no. (1-436-4606). The authors, therefore, acknowledge technical and financial support of Taif University. The support is in the form of project for academic research at TU. 
Figures

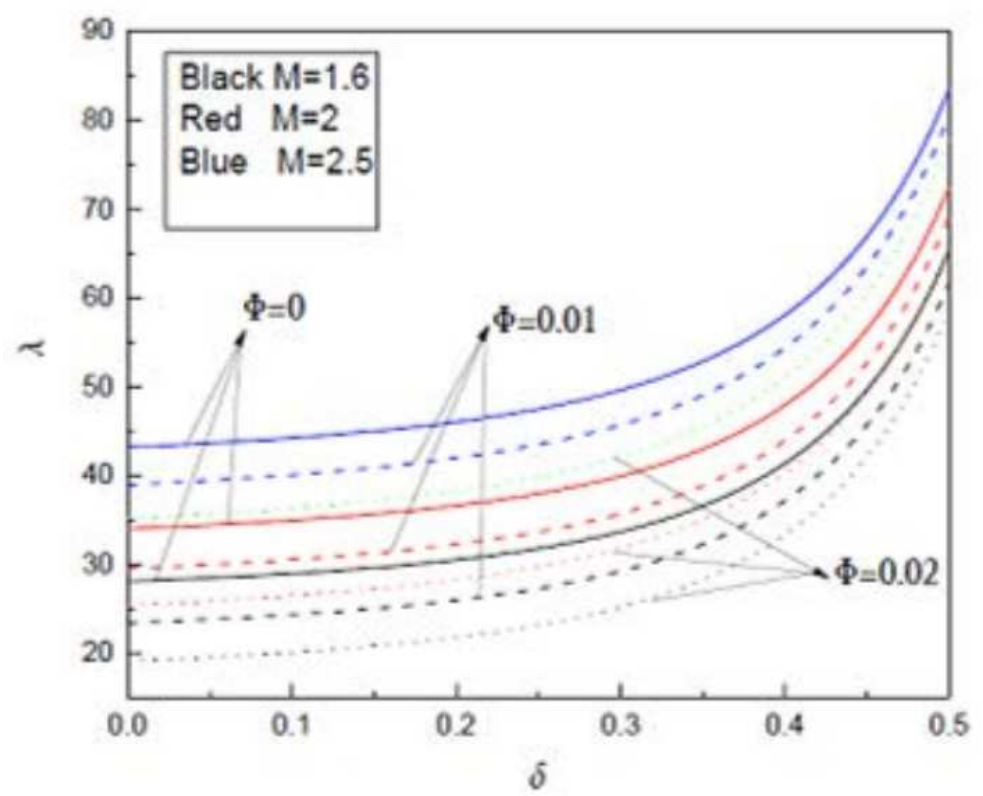

Figure 2: Variation of resistance impedance for different values of $M$ and $\Phi$, other parameters are $\mathrm{G}_{\mathrm{r}}=1, \mathrm{~F}=0.1, \beta=0.15$. 


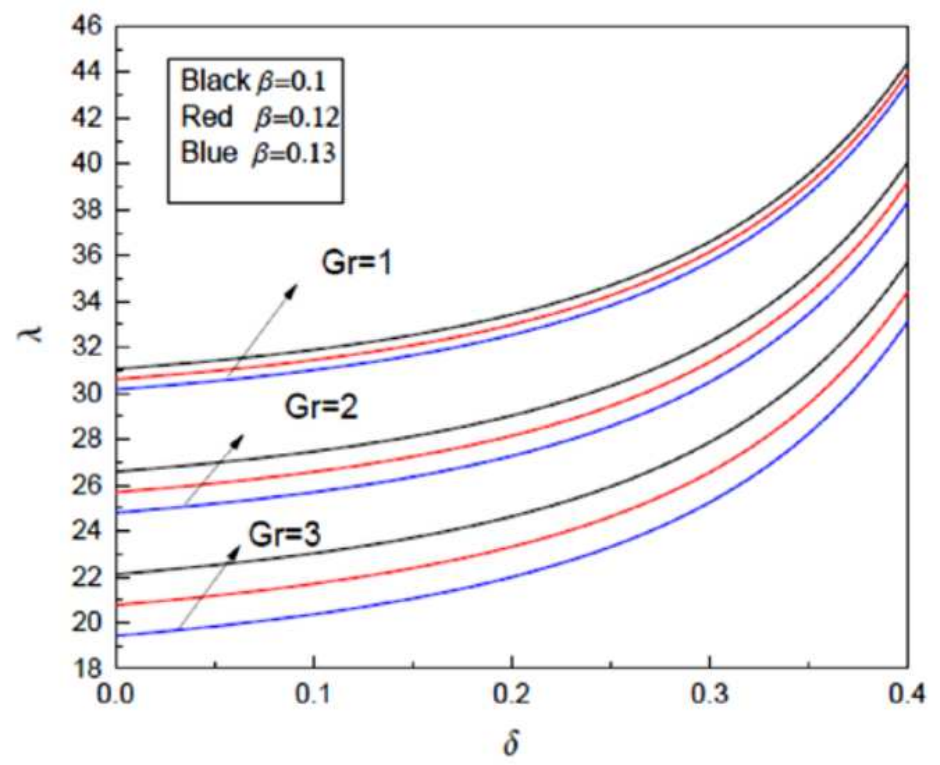

Figure 3: Variation of resistance impedance $\lambda$ for different values of $\beta$ and $\mathrm{G}_{\mathrm{r}}$, other parameters are $M=1, \Phi=0.01, \mathrm{~F}=0.1$.

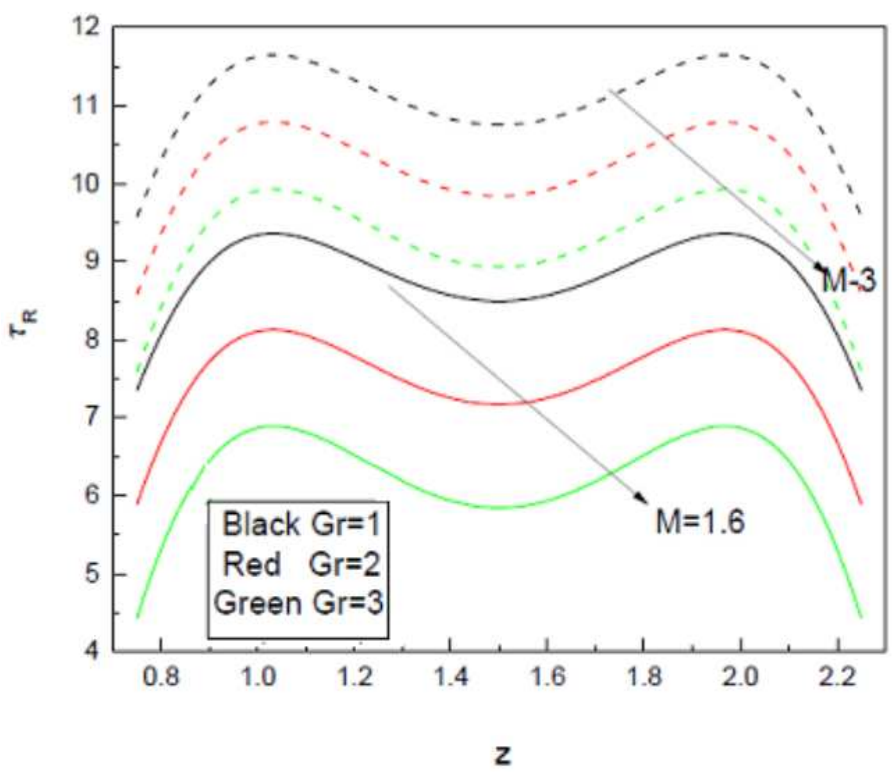

Figure 4: Variation of shear stress distribution $\tau_{\mathrm{R}}$ for different values of $\mathrm{M}$ and $G r$, other parameters are $\delta=0.1, \beta=0.1, \mathrm{~F}=0.1, \Phi=0.01$. 


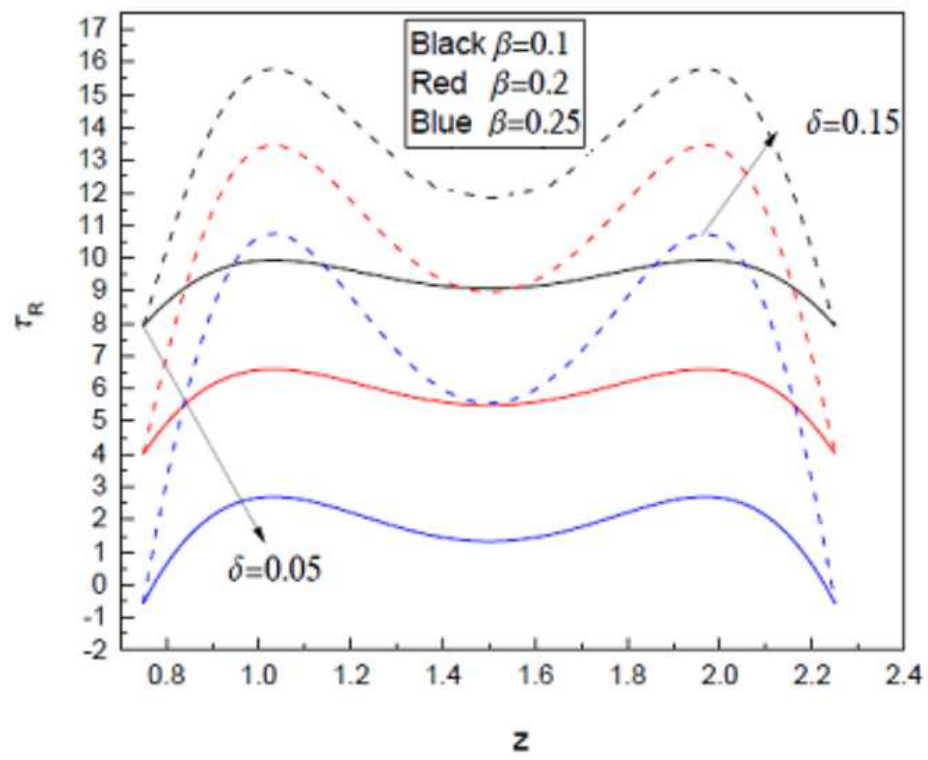

Figure 5: Variation of shear stress distribution $\tau_{\mathrm{R}}$ for different values of $\beta$ and $\delta$, other parameters are $\mathrm{G}_{\mathrm{r}}=0.1, \mathrm{M}=1.5, \mathrm{~F}=0.1, \Phi=0.01$.

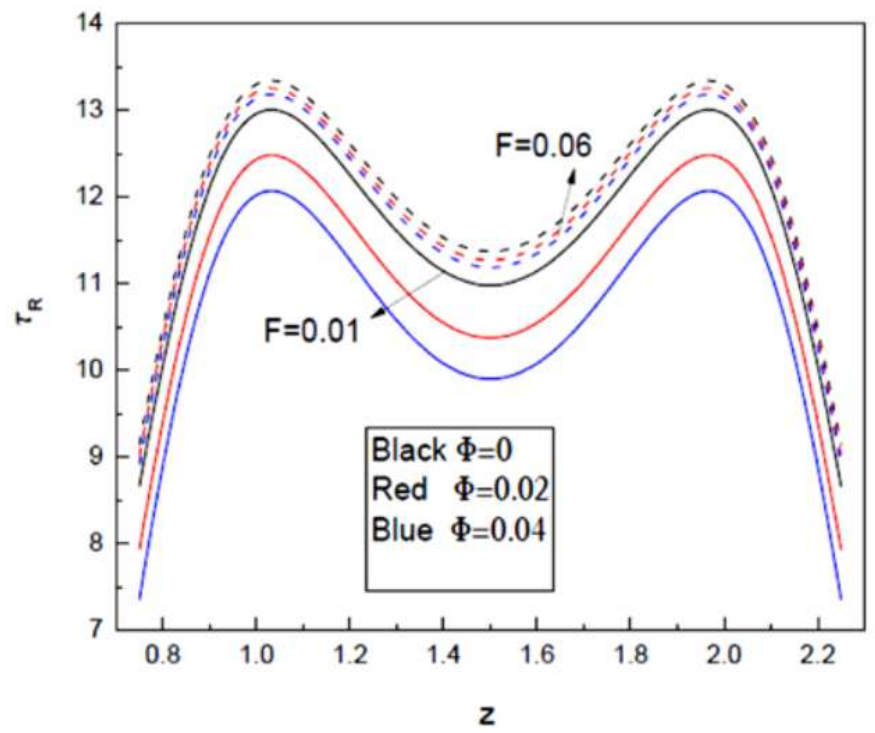

Figure 6: Variation of shear stress distribution $\tau_{\mathrm{R}}$ for different values of $\mathrm{F}$ and $\Phi$, other parameters are $\mathrm{G}_{\mathrm{r}}=0.1, \mathrm{M}=1.5, \beta=0.1, \delta=0.01$. 


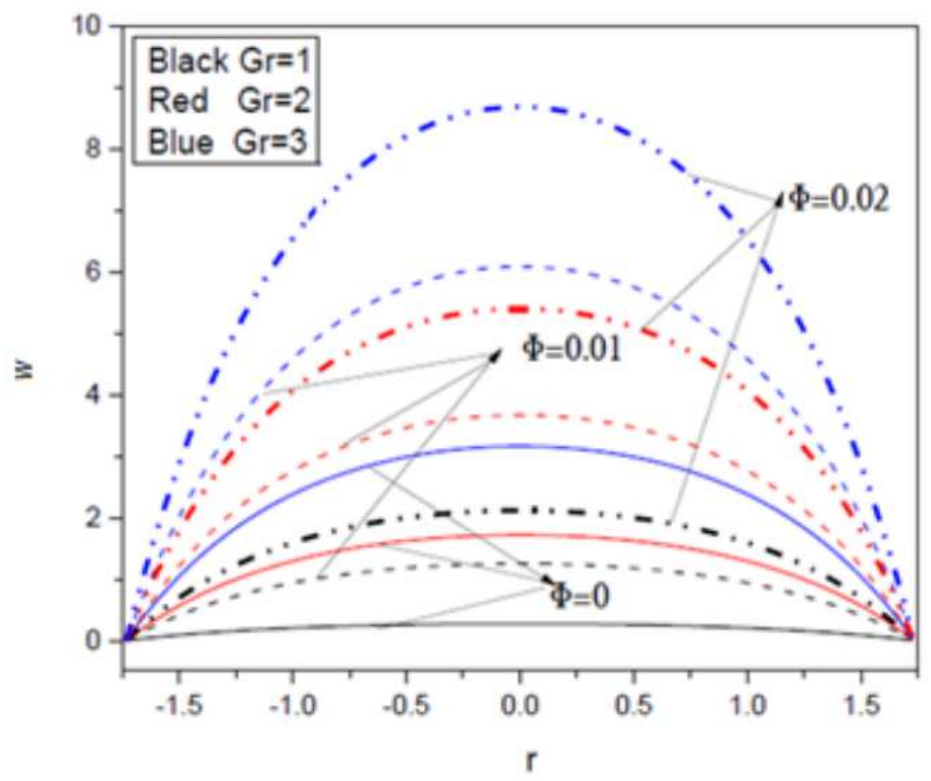

Figure 7: Variation of velocity distribution w for different values of $\mathrm{Gr}$ and $\Phi$, other parameters are $\mathrm{F}=0.1, \mathrm{M}=1.5, \beta=0.1, \delta=0.05$. 


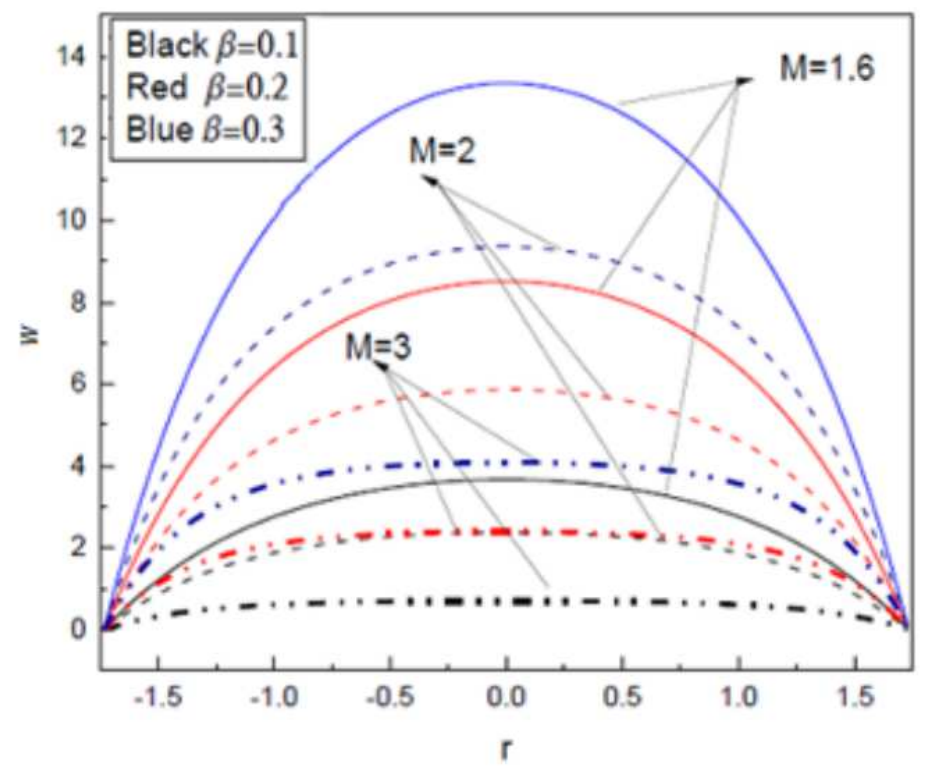

Figure 8: Variation of velocity distribution $\mathrm{w}$ for different values of $\beta$ and $\mathrm{M}$, other parameters are $\mathrm{F}=0.1, \Phi=0.01, \mathrm{Gr}=0.1, \delta=0.05$.

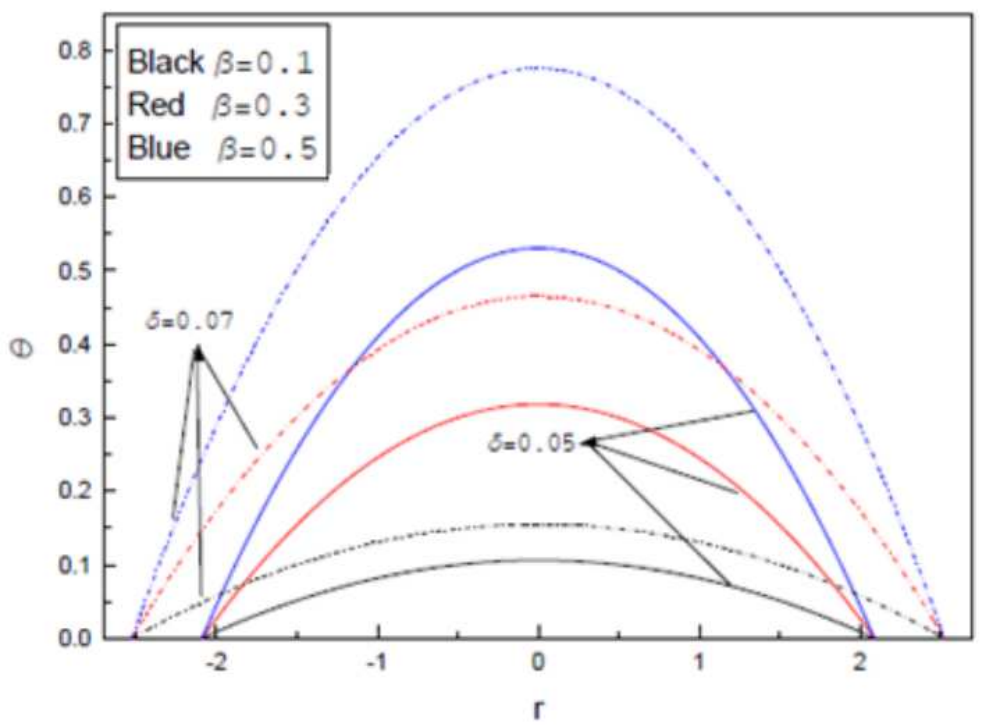

Figure 9: Variation of temperature distribution $\Theta$ for different values of $\beta$ and $\delta$, other parameters are $\mathrm{F}=0.1, \mathrm{M}=1.5, \mathrm{Gr}=0.1, \Phi=0.01$. 


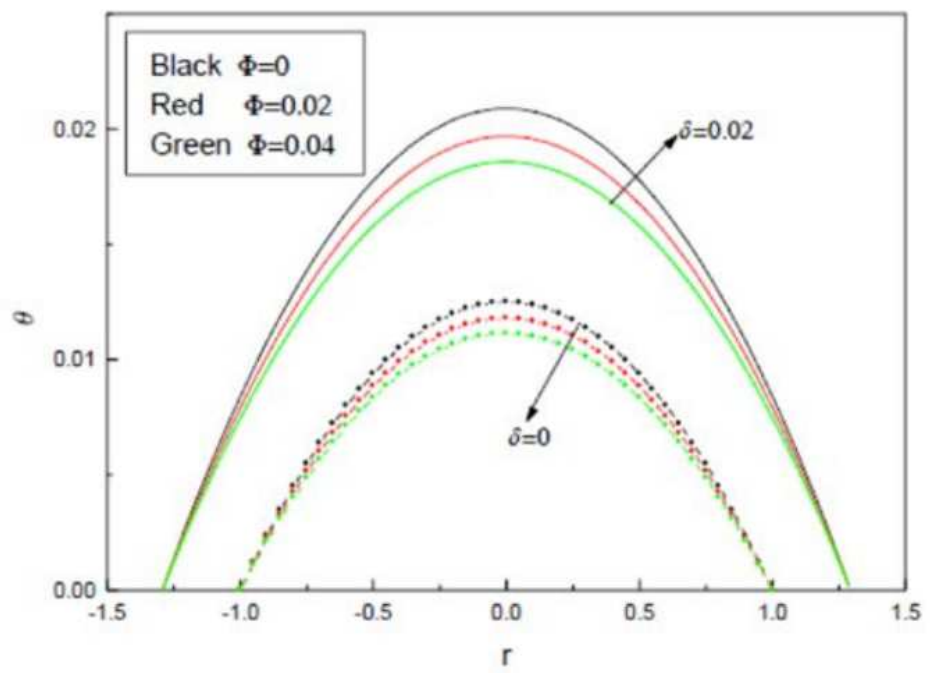

Figure 10: Variation of temperature distribution $\Theta$ for different values of $\Phi$ and $\delta$ other parameters are $\mathrm{F}=0.1, \mathrm{M}=1.5, \mathrm{Gr}=0.1, \beta=0.1$.

(a)

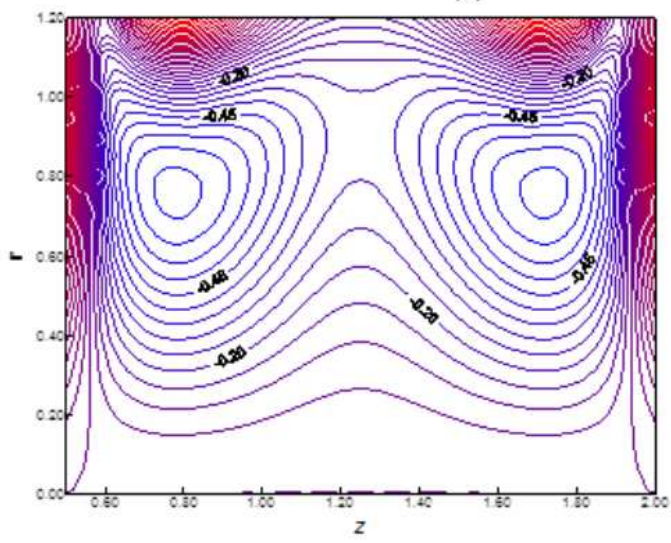

(b)

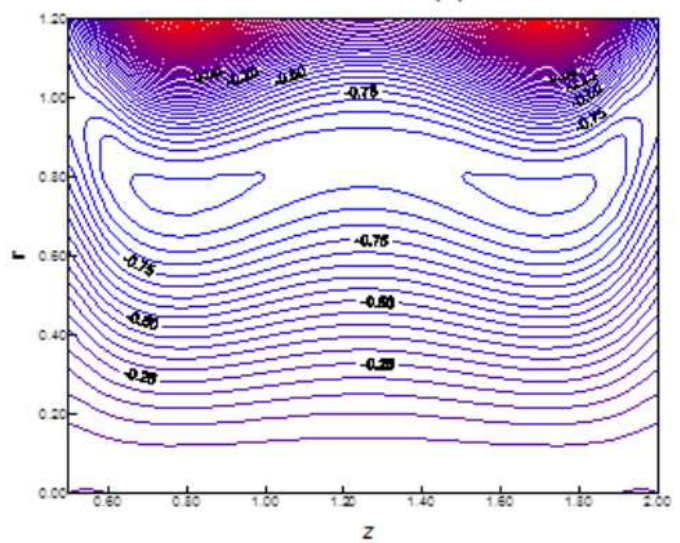

Figure 11: Fluid flow pattern for (a) $\beta=0.05$, (b) $\beta=0.5$ and other parameters are $\mathrm{F}=0.01, \mathrm{M}=1.5, \mathrm{Gr}=2, \Phi=0.02, \delta=0.2$. 
(a)

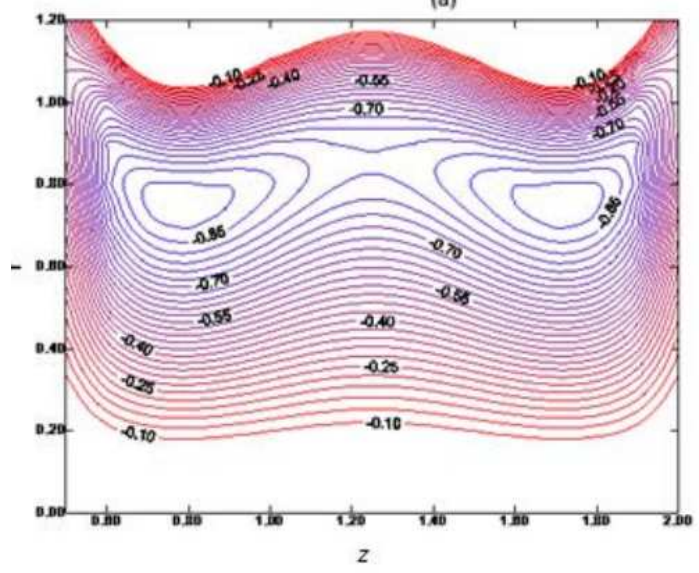

(b)

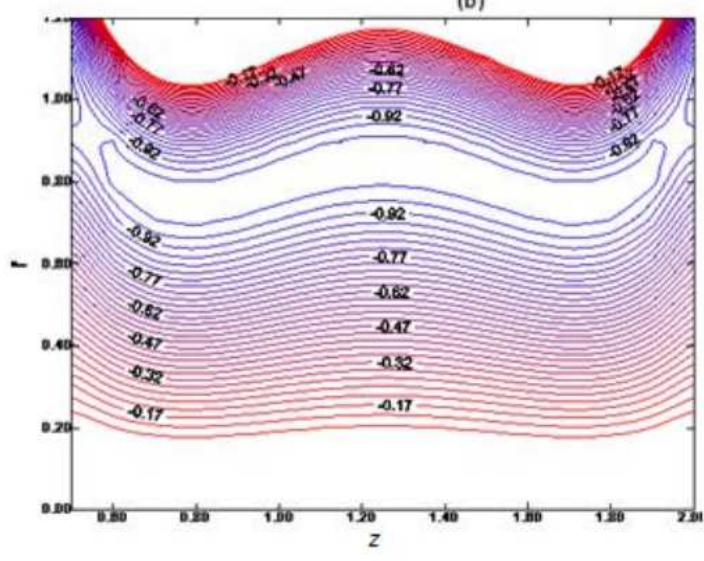

Figure 12: Fluid flow pattern for (a) $\mathrm{Gr}=1$, (b) $\mathrm{Gr}=3$ and other parameters are $\mathrm{F}=0.01, \mathrm{M}=1.5, \beta=0.05, \Phi=0.02, \delta=0.2$.

(a)

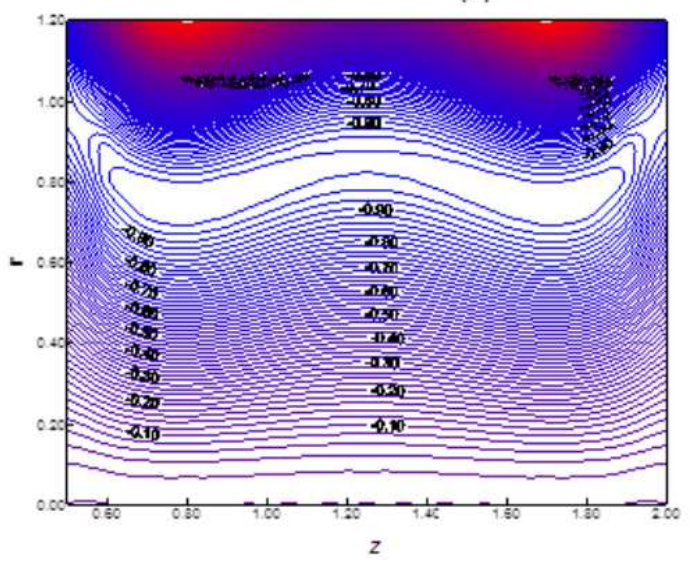

(b)

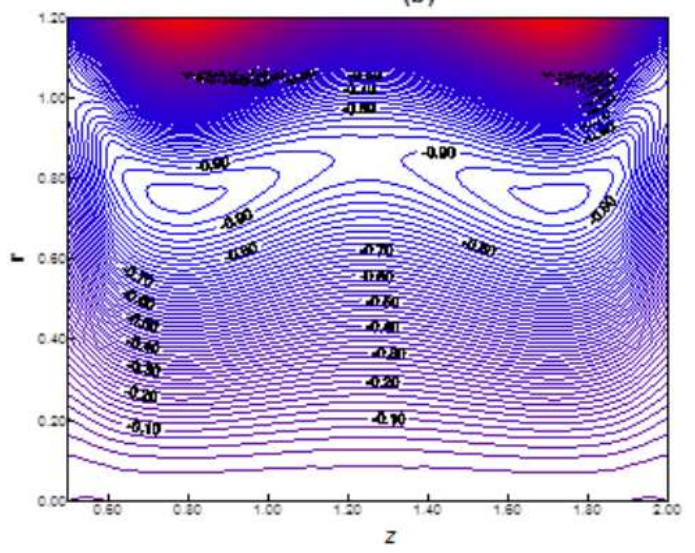

Figure 13: Fluid flow pattern for (a) $\mathrm{F}=0.01$, (b) $\mathrm{F}=0.3$ and other parameters are $\mathrm{Gr}=2, \mathrm{M}=1.5, \beta=0.05, \Phi=0.02, \delta=0.2$. 


\section{References}

[1] S. Chakravarty, and A. G. Chowdhury, Response of blood flow through an artery under stenotic conditions. Rheol Acta, 27 (1988)., 418-427

[2] Kh S. Mekheimer, M. H. Haroun, and M. A. Elkot, Induced magnetic field influences on blood flow through an anisotropically tapered elastic arteries with overlapping stenosis in an annulus. Can. J. Phys, 89 (2011), 201-212.

[3] I. J. D Craig. and P. G.Watson, Magnetic reconnection solutions based on a generalized Ohm's law. Solar Physics, 214 (2003), 131-150

[4] V. K.Stud, G. S.Sephon, and R. K. Mishra, Pumping action on blood flow by a magnetic field. Bull. Math. Biol., 39 (1977), 385

[5] H. L. Agrawal, and B.Anwaruddin, Peristaltic flow of blood in a branch. Ranchi Univ. Math. J., 15 (1984), 111

[6] M. A. A. Elnaby, and M. H. Haroun, A new model for study the effect of wall properties on peristaltic transport of a viscous fluid. Communications in Nonlinear Science and Numerical Simulation, 13 (2008), 752-762

[7] M. H. Haroun, On non-linear magnetohydrodynamic flow due to Peristaltic transport of an oldryd 3-constant fluid. Z. Naturforsch, 61a (2006), 263-274

[8] M. H Haroun, Non-linear Peristaltic flow of a fourth grage fluid in an inclined asymmetric channel.Computational Material Science, 39 (2007), 324-333

[9] Kh. S.Mekheimer and M. A.Elkot, The micropolar fluid model for blood flow through stenotic arteries. Int. J. pure and App. Math., 4 (2007), 393-405

[10] Kh. S.Mekheimer, and M. A. Elkot, Influence of Magnetic field and Hall currents on blood flow through stenotic artery. Appl. Math. Mech. Engl. Ed., 29(8), 1093-1104 (2008),DOI.10.1007/s10483-008-0813-x

[11] Kh. S. Mekheimer, and M. A. Elkot, The micropolar fluid model for blood flow through a tapered arteries with a stenosis. Acta Mech. Sin., 24 (2008), 637-644

[12] Kh. S. Mekheimer, and M. A. Elkot, Suspension model for blood flow through arterial catheterization. Chem. Eng. Comm., 197(2010), 1-20

[13] D.Biswas, and U. S.Chakraborty, Pulsatile blood flow through a catheterized artery with an axially nonsymmetrical stenosis. Applied Mathematical Sciences, 4(58) (2010), 28652880

[14] V.P. Srivastava, R. Rastogi, and R.Vishnoi, A two-layered suspension blood flow through an overlapping stenosis. Computers and Mathematics with Applications, 60(2010), 432441

[15] D. S. Sankar. and U. Lee, Two-fluid Casson model for pulsatile blood flow through stenosed arteries: A theoretical model. Commun. Nonlinear Science. Numerical. Simulat., 15(2010), 2086-2097,

[16] V. P. Srivastava, S. Mishra, and R. Rastogi, Non-Newtonian arterial blood flow through an overlapping Stenosis. A. A. M. International Journal, 5(1) (2010), 225-238

[17] S. U. Siddiqui, N. K Verma, S. Mishra, and R. S. Gupta, Mathematical modelling of pulsatile flow of Casson,s fluid in arterial stenosis. Applied Mathematics and Computation, 210(2009), 1-10 
[18] G. C. Layek, Mukhopadhyay, S., and Gorla, R. S. R. Unsteady viscous flow with variable viscosity in a vascular tube with an overlapping constriction. International Journal of Engineering Science, 47(2009), 649-659

[19] B. Pincombe, J. Mazumdar, and I. Hamilton-Craig, Effects of multiple stenoses and post-stenotic dilatation on non-Newtonian blood flow in small arteries. Medical Biological Engineering Computing, 37(1999), 595-599

[20] B. Tashtoush, and A. Magableh, Magnetic field effect on heat transfer and fluid flow characteristics of blood flow in multi-stenosis arteries. Heat Mass Transfer, 44(2008), 297-304

[21] M. X. Li, Beech-Brandta, J. J., Johnb, L. R., Hoskinsc, P. R., and Eassona, W. J. Numerical analysis of pulsatile blood flow and vessel wall mechanics in different degrees of stenoses. Journal of Biomechanics, 40(2007), 3715-3724

[22] B. K. Mishra and Verma, N. Magnetic effect on blood flow in a multiple stenosed artery. Applied Mathematics and Computation, 1(2007), 1-7

[23] S. U. S. Choi, Enhancing thermal conductivity of fluids with nanoparticles, In: Siginer DA, Wang HP (eds), Developments and applications of non-Newtonian flows, ASME,(1995), 36- 99.

[24] S. Nadeem, R. U. Haq, Z. H. Khan, Heat transfer analysis of water-based nanofluid over an exponentially stretching sheet, Alexandria Engineering Journal, (2014), 53- 219.

[25] N. S. Akbar, Peristaltic flow of Cu-water nanofluid in a tube, Journal of computational and theoretical nanosciences, (2014), 11- 1411.

[26] N. S. Akbar, E. N. Maraj, S. Nadeem, Copper nanoparticle analysis for peristaltic flow in a curved channel with heat transfer characteristics, Eur. Phys. J. Plus, (2014), 129-149.

[27] J. Buongiorno, Convective transport in nanofluids, J. Heat Transfer (Amer. Soc. Mech.Eng.), (2005), 128- 240.

[28] S. Nadeem, S. Ijaz and N. S. Akbar, Nanoparticle analysis for blood flow of Prandtl fuid model with stenosis, International Nano Letters, (2013), doi:10.1186/2228-5326-3-35.

[29] N. S. Akbar, Z. H. Khan, Heat transfer study of an individual multiwalled carbon nanotube due to metachronal beating of cilia, International Communications in Heat and Mass Transfer, (2014), 59- 114.

[30] N. S. Akbar, E. N. Maraj, S. Nadeem, Copper nanoparticles impinging in a curved channel with complaint walls and peristalsis, Eur. Phys. J. Plus, (2014), 129- 183.

[31] N. S. Akbar, CNT suspended nanofluid analysis in a flexible tube with ciliated walls, Eur. Phys. J. Plus, (2014), 129-174. 